\title{
Miranda
}

Revue pluridisciplinaire du monde anglophone /

Multidisciplinary peer-reviewed journal on the English-

speaking world

$14 \mid 2017$

Early American Surrealisms, 1920-1940 / Parable Art

\section{Pierre Morère, Sens et sensibilité: pensée et poésie dans la Grande-Bretagne des Lumières}

\section{Marc Porée}

URL : http://journals.openedition.org/miranda/10026

DOI : 10.4000/miranda.10026

ISSN : 2108-6559

Éditeur

Université Toulouse - Jean Jaurès

Référence électronique

Marc Porée, «Pierre Morère, Sens et sensibilité : pensée et poésie dans la Grande-Bretagne des

Lumières », Miranda [En ligne], 14 | 2017, mis en ligne le 03 avril 2017, consulté le 16 février 2021. URL : http://journals.openedition.org/miranda/10026 ; DOI : https://doi.org/10.4000/miranda.10026

Ce document a été généré automatiquement le 16 février 2021.

\section{(c) (i) () $\Theta$}

Miranda is licensed under a Creative Commons Attribution-NonCommercial-NoDerivatives 4.0 International License. 


\title{
Pierre Morère, Sens et sensibilité : pensée et poésie dans la Grande- Bretagne des Lumières
}

\author{
Marc Porée
}

\section{RÉFÉRENCE}

Morère, Pierre, Sens et sensibilité : pensée et poésie dans la Grande-Bretagne des Lumières (Lyon : Presses universitaires de Lyon, coédition ELLUG-PUL, collection « Esthétique et représentation : monde anglophone, (1750-1900) », 2015), 340 p, ISBN-13 :

978-2-7297-0895-5

1 Il faut savoir gré à Pierre Morère, professeur émérite à l'Université Stendhal-Grenoble 3 , de nous rappeler avec force quelques évidences oubliées. Non, le dix-huitième siècle britannique ne fut pas le seul siècle des romanciers. Il fut aussi le siècle des poètes, et des grands poètes, par dessus le marché : Pope, Cowper, Thomson, mais encore Smart, Rochester, Young, Blake, Beattie (dont Morère est un grand spécialiste), et bien sûr William Wordsworth, sur la fin de la période. Son ouvrage, sobrement intitulé Sens et sensibilité, entreprend une réhabilitation en bonne et due forme des conceptions et des réalisations poétiques d'un siècle présenté à tort comme hostile à la poésie. Il n'en fut bien sûr rien. De surcroît, et là encore notre gratitude envers lui est grande, Morère s'efforce de rétablir dans ses droits ce qui est ordinairement présenté, au mieux ou au pire, c'est selon, comme relevant d'un seul préfixe opportun : le pré de pré-romantisme. Ne voulant pas céder à la tentation téléologique qui préside souvent aux histoires de la littérature, laquelle gauchit la perspective en la subordonnant à un devenir perçu a posteriori comme inéluctable, Morère refuse d'escamoter tout le long amont du romantisme, au motif qu'il n'aurait existé que pour mieux (s') y préparer, pour en être le « Prélude». 
2 Rien de tel ici; la poésie du dix-huitième siècle anglais ne fut ni une antichambre ni même un long vestibule, tout au plus un "sofa » sur lequel elle se sera étendue, et encore, jamais mollement, mais plutôt ardemment et conceptuellement, le plus souvent. Venue de loin, nourrie de son fonds propre, elle n'aura pas été propédeutique ni même liminaire, sauf en toute fin de parcours, avec les Ballades lyriques de Wordsworth et de Coleridge. Non, elle fut pleinement elle-même, existant à part entière, sans rien d'ancillaire ni de subordonné, et c'est d'un regard dépouillé de toute perspective cavalière, et a fortiori fuyante ou dépravée, que Morère envisage les liens unissant la pensée des Lumières et la poésie, faisant de cette dernière la résultante d'une dialectique, autant bien tempérée que bien trempée, entre foi et ferveur, tempérance et excès, réalisme et imagination, réalité et rêve, réel et surréel. Non, encore, elle ne fut pas placide ou complaisamment ordonnée: Morère souligne, à l'occasion, les désordres, les sources d'aliénation ou d'angoisse, les questions sur le mystère de l'au-delà restées sans réponses.

3 Divisant son riche matériau en deux plans - le plan des " prolégomènes ", conceptuels suivi du plan des "phénomènes" pour adopter le vocabulaire philosophique d'un Emmanuel Kant -, Morère en profite pour batailler contre le classicisme français, et les Lumières françaises (encore que leurs philosophes soient assez peu convoqués). Avisé autant que perspicace, il pointe la montée en puissance du sensualisme, tout en ne cachant rien de ses limites, ni des intuitions supra-sensorielles qui se firent jour chez les poètes, plus souvent qu'à leur tour, d'ailleurs.

4 Sans le dire ouvertement, encore, Morère ambitionne, après d'autres, de corriger le strabisme divergent d'un T.S. Eliot, quand ce dernier pointait la soi-disant funeste dissociation of sensibility survenue après Milton, à l'origine d'une impossibilité pour les poètes postérieurs de penser et de sentir simultanément. C'est au contraire une sensibilité unifiée, pleinement réconciliée, à l'unisson, qu'il nous présente, par le prisme de laquelle, assurément, les poètes surent penser et sentir in the same breath. Autre redressement ou correctif: le primat du sensible, dont Morère entreprend de remotiver les potentialités poétiques. Et s'il ne parle pas, stricto sensu, de «partage du sensible », pour reprendre les termes de Jacques Rancière, il n'en omet pas moins de relever toutes les implications, pour les lecteurs de poésie, d'une telle orientation. En définitive, c'est par un chiasme parfait qu'il cadre son propos : la poésie des Lumières sera passée d'un empirisme ouvert et d'un sensualisme étroit à un sensualisme ouvert prenant le pas sur un «empirisme restrictif» (p. 308). Soit les trois moments d'une dialectique ainsi résumée: "une première phase normative encore inspirée des classiques, une étape réceptive fondée sur les données du sensualisme, et une amorce de synthèse entre création et réception énoncée dans les préfaces des Lyrical Ballads» (p. 306).

5 On le voit, la démarche est celle d'un chercheur, qui s'emploie à placer sa démarche sous le signe, précisément, d'une « recherche » (bien plus que d'une quête). L'influence de la philosophie du temps, avec ses nombreuses Enquiries, y est pour beaucoup. «Une poétique de la recherche ", nous est-il dit en quatrième de couverture : procédant avec méthode et détermination - deliberately, aurait dit Samuel Johnson, le "Great Cham » - Morère développe son programme, lequel suit très exactement l'ordre des mots avancés dans son titre et son sous-titre, à savoir "sens et sensibilité », d'une part, "pensée et poésie » de l'autre. Où l'on comprend que la pensée précède la poésie, tout en lui étant étroitement associée. C'est donc principalement en historien des idées que 
Morère analyse l'effervescence intellectuelle du temps, laquelle s'exprime en autant de "mécanismes mentaux ", lesquels s'avèrent déterminants pour expliquer en quoi ils «ouvrent aux poètes les voies du sensible» (p. 11) ; sont ainsi passés en revue les philosophes et les penseurs, de Locke à Hume, en passant par Berkeley, Shaftesbury, Adam Smith et Burke. Locke s'y taille la part du lion, à juste titre, mais le scepticisme de Hume n'est pas absent du propos, pas plus que son relativisme, qui revient à situer le goût dans la sphère du purement subjectif ; c'est même sa sociologie de l'esthétique qui permet de mettre en avant « le contexte dans lequel les arts peuvent s'épanouir, c'està-dire une dialectique du beau et de l'utile» (p. 287).

6 Tout en accordant la priorité aux systèmes de pensée, Morère n'en oublie pas de prendre en charge des objets plus transversaux, tels que la pensée du bonheur, thème central de la pensée des Lumières, la pensée de la nature, de l'élan vital (voir la confiance placée par Pope dans les instincts, dont Morère nous rappelle combien elle s'explique, malgré son caractère "inattendu »), la pensée de l'homme, dont l'Essay on Man du même Pope est un sommet auquel sont consacrées une bonne trentaine de pages denses et éclairantes. Aux côtés de la pensée du relatif, la question du théisme, tout aussi centrale, est traitée de la plus convaincante des manières. Reste la pensée de la poésie, ou à tout le moins du poétique, que Morère aborde sous ses angles essentiellement critique et théorique. "Le poème est-il déterminé par le paradigme du sens ou par les réalité empirique, écrasante, de la sensibilité ?» (p. 13); " en quoi l'œuvre versifiée est-elle de nature transcendante?" (p. 13). Telles sont les deux interrogations majeures qui traversent le livre, et c'est à la lumière des réponses qui leur furent apportées que Morère se fait fort de recontextualiser ce qu'il nomme le «site du poétique » (p. 13).

7 Plus précisément, Pierre Morère se veut à la recherche de ce qui engage la réflexion sur des voies nouvelles, lesquelles avaient en partage la connaissance des mondes extérieurs et intérieurs, tout comme l'apparition de nouvelles sources d'inspiration dans le domaine de la poésie (p. 264). Au fil des décennies, celles-ci s'affirmeront. Rétrospectivement, on y aura perçu l'absolutisation croissante du poétique et du littéraire en tant que tel, la montée en puissance de la critique comme genre à part entière, l'assomption du moi, du Logos et de la subjectivité, les émois et les transports du corps, l'ébranlement provoqué par la Révolution française - de quoi faire céder les dernières digues retenant ce qui n'était pas, à dire vrai, le siècle de la raison, mais bien celui, la distinction est de taille, celui des « sens régulés par le jugement» (p. 62). C'est dire si, pour l'exprimer autrement, le glissement progressif et résolu vers l'imagination y était dès le début inscrit en germe...

8 Conduit de main de maître, l'ouvrage fait toucher du doigt à chaque instant la nature particulière de la réflexion qui animait penseurs et poètes, quasiment envisagée dans ce qui en constituait le grain, et en tout cas les moindres nuances. Solidement charpentée, rédigée avec une clarté exemplaire, l'enquête fera date par sa volonté de viser l'équilibre et d'aller au fond des choses, au cœur de ce qui est perçu comme l'interpénétration, finalement heureuse, du sensible, du pensif ou du spéculatif, et du poétique. Rien n'y est bousculé, et c'est de façon en tout point mesurée que Morère procède ; il suffit de se reporter aux nombreux index, et en particulier à celui des noms propres et des œuvres, pour mesurer sur pièces la place conséquente prise, respectivement, par chacun des protagonistes du temps : les dosages y sont effectués au trébuchet, confirmant ainsi l'intuition selon laquelle penser, c'est peser, et 
inversement. Certes, on pourra toujours discuter de certaines attributions ou proportions: les 5 pages dévolues à Blake pèsent sans doute peu au regard des 40 dévolues à Addison ou même des 15 affectées à Thomson. Mais ce que Morère écrit des flamboiements et autres ruptures assumées par le premier sont profondément justes, même s'il fut sans l'ombre d'un doute bien davantage qu'une «exception » qui confirme la règle, et il n'y a pas grand-chose à redire de sa compréhension de la place, relative, prise par chacune des œuvres selon une perspective qui se veut contemporaine à elles.

Doté d'un sens très sûr de la formule, dont il n'abuse cependant pas, l'auteur avance avec calme et méthode et c'est tout aussi posément qu'il conclut, en ouvrant sur le dépassement des sens (déjà mis en œuvre par Blake, fera-t-on tout au plus observer) et l'aspiration à la transcendance qui seront l'apanage du romantisme. Chemin faisant, Morère n'aura rien caché du caractère à la fois proliférant et « disparate ", pour ne pas dire inégal, des œuvres poétiques dont il n'aura retenu que les plus saillantes. La partialité d'un Johnson, avec son anglo-centrisme et son néo-classicisme résolument tory, n'échappe pas non plus au regard justement critique de l'auteur, dont on connaît par ailleurs le fort tropisme calédonien (à cet égard, les sections consacrées aux penseurs écossais comptent parmi les plus stimulantes du livre). C'est du reste au même Samuel Johnson que Morère a dernièrement consacré un autre ouvrage, sous la forme d'une traduction de ses Lives of English Poets, en collaboration avec son collègue de Grenoble, le romanticiste et poéticien Denis Bonnecase. ${ }^{1}$ On voit et on salue la cohérence du propos, qui revient à faire rimer, en toutes circonstances, critique et vérité.

Deux motifs d'étonnement, pour finir, même s'il convient d'en relativiser la portée. Jane Austen, qui prête pourtant son titre à l'ouvrage dont il a été question, n'y est jamais nommée en tant que telle. Il est vrai qu'elle ne fut ni poète ni penseuse. Quant à la belle illustration de couverture, représentant une chouette effraie, non seulement on la doit à un Français, Robert Nicolas, peintre, dessinateur et graveur de son état, mais encore ce dernier exerçait-il ses talents au XVIIe siècle. Il fallait donc que le choix d'un « hybou de campagne ", selon la graphie du temps, s'imposât impérieusement.

Point n'est besoin d'aller chercher bien loin le pourquoi d'un tel choix: emblème de la sagesse, donc de la philosophie, la chouette prend son envol au crépuscule, quand retombe l'agitation de la journée. Oiseau de nuit, sa forte et éclatante présence suggère de manière assurément subliminale que les Lumières britanniques étaient travaillées, de loin en loin, par leur envers nocturne, obscurément intérieur et rapace. L'un des grands mérites de l'ouvrage de Pierre Morère consiste, de fait, dans sa très perspicace élucidation de ce qui fut tout à la fois un besoin de sens commun et une volonté de composer avec les sens, avec le corps sensible, en privilégiant pour ce faire les voies et les moyens propres à la poésie. Quitte à ce que cette dernière, effraie décidément plus singulière que commune, s'affranchisse, in fine, d'un tel programme, et achève de prendre son envol, non sans avoir troqué la sensibilité contre la sensation. Mais ceci est une autre histoire... 


\section{NOTES}

1. Samuel Johnson Vies des poètes anglais. Choix de textes, traduction et présentation de Denis Bonnecase et Pierre Morère. Paris : Librairie du Sandre, 2016.

\section{INDEX}

Mots-clés : sens, sensibilité, sensualisme, poésie, imagination, philosophie, ferveur, connaissance, néoclassicisme, lumières, esthétique

Keywords : sense, sensibility, sensualism, poetry, imagination, philosophy, fervour, knowledge, neoclassicism, enlightenment, aesthetics

\section{AUTEURS}

\section{MARC PORÉE}

Professeur des Universités

ENS-PSL, Prismes EA 4398 